\title{
Recherche des formes optimales d'alimentation des machines à réluctance variable
}

\author{
C. Glaize
}

L.E.SI.R. E.N.S.E.T. de Cachan, 61, avenue du Président Wilson, 94230 Cachan, France

(Reçu le 22 avril 1985, révisé le 11 juillet, accepté le 16 juillet 1985)

\begin{abstract}
Résumé. - Dans cet article, l'auteur se propose de rechercher des formes de courant d'alimentation de machines à réluctance variable permettant d'en optimiser le fonctionnement. Après une présentation des critères d'optimisation, on calcule les formes d'ondes optimales et le couple obtenu pour divers types de machines en non-saturé, puis en saturé. Les incidences sur l'ondulation du couple et le nombre de phases optimal sont aussi étudiées. Enfin est présenté le schéma d'un convertisseur délivrant simplement quelques formes d'ondes quasi optimales.
\end{abstract}

\begin{abstract}
In this paper, the author proposes to search for forms of supply current for reluctance machines allowing their optimization. After the optimization criteria have been introduced, the optimal waveforms and the resulting torque are calculated for different types of machines in non-saturated area then in saturated area. The incidence on the torque modulation and the optimal number of phases are also studied. Finally, the scheme of a converter which delivers a few quasi-optimal waveforms is presented.
\end{abstract}

Pour l'alimentation des machines à réluctance variable, on peut chercher, au-delà des alimentations sinusoïdales ou rectangulaires de tension ou de courant, s'il existe d'autres formes d'ondes qui améliorent le fonctionnement de la machine ou du convertisseur. C'est sur ce thème que travaillent des chercheurs du LESIR (ENSET de Cachan) et du Laboratoire d'Electrotechnique des Universités Paris VI et XI. L'étude proposée ici a débuté sur les machines à réluctance variable polydiscoïdes étudiées dans ce dernier laboratoire. Mais, les calculs sont développés de manière à ce que les résultats puissent être applicables, non seulement à ce type de machines, mais aussi à d'autres géométries de machines à réluctance variable. Ils sont effectués en valeurs instantanées plutôt qu'en valeurs moyennées pour tenir compte de la spécificité de chaque machine.

Le travail décrit ici s'inscrit dans un contexte plus général d'étude et d'optimisation de machines à réluctance variable polydiscoïdes proposé par $\mathrm{C}$. Rioux [1]. Après les études de faisabilité [2], de calcul de la forme des plots (dents) magnétiques $[3,4]$, il était nécessaire de se préoccuper de manière approfondie de l'alimentation de telles machines.

\section{Modélisation de la machine.}

Vue de ses bornes d'alimentation, une machine à réluctance variable peut être considérée comme une inductance fonction, d'une part, de la position angulaire du rotor par rapport au stator (effet de réluctance variable) et, d'autre part, du courant (effet de saturation). Pour être plus général, on remplace l'inductance qui dépend du nombre de spires d'un enroulement, par la perméance (notée $z$ ) qui est l'inverse de la réluctance. De même, on remplace le courant par les ampères-tours créés (notés $j$ ).

Une machine à réluctance variable peut prendre de multiples formes géométriques donnant des fonctions de perméance variées [5-9]. Il est cependant possible de raisonner sur un modèle bipolaire cylindrique simplifié (Fig. 1). En particulier, on utilise la notion d'angle électrique $\theta$, produit de l'angle mécanique par le nombre $\boldsymbol{P}$ de plots (ou de dents) de la machine. Sur le modèle simplifié apparaissent clairement les positions dites de conjonction $(\theta=\pi)$ pour laquelle les dents fixes ou mobiles sont en vis-à-vis et celles dites d'opposition $(\theta=0)$ pour laquelle la réluctance est maximale. 


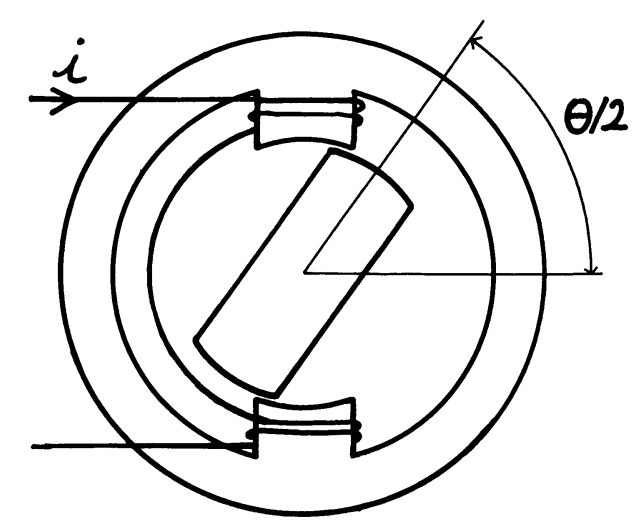

Fig. 1. - Modèle simplifié de machine à réluctance variable. [Simplified scheme of a reluctance machine.]

Dans une première étape, nous ne nous sommes intéressés qu'aux machines polyphasées sans couplage magnétique entre phases. Ces dernières peuvent être constituées d'un empilement de machines monophasées convenablement décalées [10]. Par souci de ne pas augmenter le nombre de paramètres d'étude, on peut négliger les pertes Joule et les pertes fer. Cela se vérifie expérimentalement sur les machines lentes de forte puissance [11]. Les fuites magnétiques sont prises en compte dans la modélisation. Elles ajoutent un terme à la perméance utile. Si les pertes fer sont négligeables, ces fuites ne provoquent aucun travail et leur contribution à la puissance moyenne est nulle. Pour le convertisseur, la machine est donc entièrement déterminée par la connaissance de la fonction perméance $z(\theta, j)$.

\section{Critères d'optimisation.}

Dans un premier temps, les deux critères d'optimisation retenus sont :

- la maximisation du couple moyen à courant crête donné,

- la maximisation du couplè moyen à courant efficace donné.

Pour la machine, le choix de la maximisation du couple moyen est équivalent à extraire le maximum de puissance à une vitesse de rotation (donc pour une fréquence d'alimentation) donnée. Le premier de ces critères correspond plutôt à une optimisation d'un convertisseur à transistors ou à thyristors en commutation forcée. Le second impose un couple moyen maximal pour des pertes Joule données dans les enroulements de la machine. Il peut être aussi utilisé dans le cas d'une alimentation par convertisseur à thyristors en commutation naturelle ou assistée.

Le couple instantané étant proportionnel au carré du courant, il est intéressant, pour obtenir des valeurs indépendantes du courant (résultats sous forme de grandeurs réduites), de remplacer les courants efficaces et crêtes par leurs carrés. De manière totalement équivalente aux critères précédents, on choisit :

- la maximisation du rapport du couple moyen au carré du courant crête,

- la maximisation du rapport du couple moyen au carré du courant efficace.

C'est-à-dire que l'on a à optimiser les rapports :

$$
\begin{aligned}
& \langle c\rangle / J_{M}^{2} \\
& \langle c\rangle / J^{2}
\end{aligned}
$$

où $\langle c\rangle$ représente le couple moyen produit par la machine et $J$ et $J_{M}$ les valeurs efficace et crête des ampères-tours $j$.

\section{Optimisation en régime non saturé.}

Hors de la zone de saturation magnétique, la perméance $z$ est indépendante des ampères-tours $j$. Elle ne dépend plus que de la position angulaire du rotor par rapport au stator $\theta$. Le couple instantané $c$ produit par une machine à réluctance variable s'écrit dans ces conditions [12] :

$$
c=\frac{P}{2} \cdot j^{2} \cdot \frac{\mathrm{d} z}{\mathrm{~d} \theta}
$$

3. 1 MAXIMISATION DU RAPPORT $\langle c\rangle / J_{M}^{2}$. - Pour chaque angle, les ampères-tours $j$ peuvent prendre une valeur quelconque entre 0 et $J_{M}$. Pour maximiser le couple moyen en fonctionnement de la machine en moteur, il est évident que $j$ doit être égal à $J_{M}$ si $\mathrm{d} z / \mathrm{d} \theta>0$ et à zéro si $\mathrm{d} z / \mathrm{d} \theta<0$. En génératrice, il faut inverser le signe du couple et $j$ doit alors être nul pour $\mathrm{d} z / \mathrm{d} \theta>0$. On peut admettre que la perméance croît continûment entre l'opposition et la conjonction pour $0<\theta<\pi$ et décroît continûment de la conjonction à l'opposition pour $\pi<\theta<2 \pi$. Cette hypothèse est pratiquement toujours vérifiée sur les machines à réluctance variable. La forme de courant optimale est donc une onde rectangulaire d'amplitude $J_{\mathrm{M}}$ pour $\mathrm{d} z / \mathrm{d} \theta>0$ en fonctionnement moteur (Fig. 2) ou pour $\mathrm{d} z / \mathrm{d} \theta<0$ en fonctionnement génératrice. Le couple moyen $\langle c\rangle$ ne dépend alors pas de la forme de la variation de $z(\theta)$ mais uniquement de ses valeurs extrêmes et vaut (en moteur) :

$$
\langle c\rangle=\frac{P}{4 \pi} \cdot J_{M}^{2} \cdot\left(Z_{M}-Z_{0}\right)=\frac{P}{4 \pi} \cdot J_{M}^{2} \Delta Z .
$$

Le rapport du couple au carré des ampères-tours crêtes s'écrit alors :

$$
\langle c\rangle / J_{M}^{2}=\frac{P . \Delta Z}{4 \pi} .
$$

3.2 MAXImisation DU RAPPORT $\langle c\rangle / J^{2}$. - Pour maximiser le couple moyen par rapport au carré des ampères-tours efficaces, on peut ici encore remarquer que les ampères-tours $j$ doivent être nuls pour $\pi<\theta<2 \pi$ en fonctionnement moteur (entre 0 et $\pi$ 


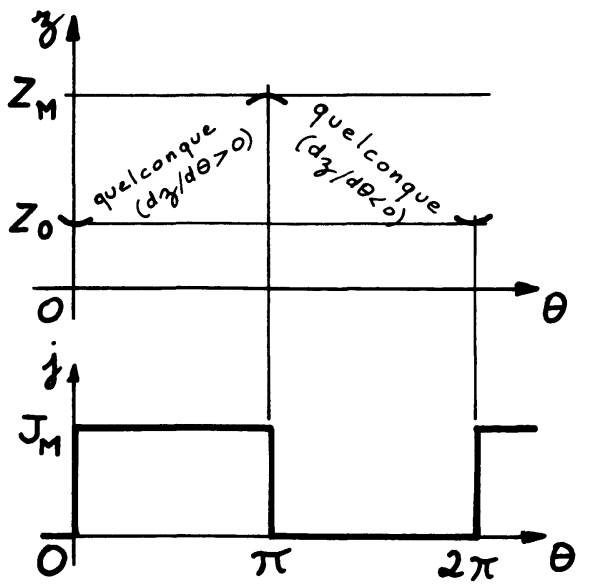

Fig. 2. - Onde de courant maximisant $\langle c\rangle / J_{M}^{2}$ en régime linéaire (fonctionnement moteur).

[Current shape maximizing $\langle c\rangle / J_{M}^{2}$ in linear domain.]

pour un fonctionnement en génératrice). Dans ces conditions, le couple moyen s'écrit :

$$
\langle c\rangle=\frac{P}{4 \pi} \cdot \int_{0}^{\pi} j^{2} \cdot \frac{\mathrm{d} z}{\mathrm{~d} \theta} \cdot \mathrm{d} \theta
$$

et le carré des ampères-tours efficaces $J$ :

$$
J^{2}=\frac{1}{2 \pi} \cdot \int_{0}^{\pi} j^{2} \cdot \mathrm{d} \theta
$$

Le critère d'optimisation choisi nous conduit à maximiser $\langle c\rangle$ à $J^{2}$ donné. $\mathrm{d} z / \mathrm{d} \theta$ joue le rôle de fonction de pondération pour la fonction $j^{2}$. Il est clair que pour maximiser $\langle c\rangle$, il faut alors concentrer la fonction $j^{2}$ là où elle a le plus fort poids, c'est-à-dire là où $\mathrm{d} z / \mathrm{d} \theta$ est maximale. Si $\mathrm{d} z / \mathrm{d} \theta$ est maximale en un point seulement (point d'inflexion sur la courbe $z(\theta)$ ), la fonction $j$ doit avoir la forme d'une impulsion de Dirac centrée sur ce point d'inflexion (Fig. 3a). $\mathrm{Si} \mathrm{d} z / \mathrm{d} \theta$ est constante et maximale sur un intervalle $\theta_{1}<\theta<\theta_{2}$, $j$ peut prendre une valeur quelconque dans toute cette plage et doit être nul à l'extérieur (Fig. 3b). Le rapport $\langle c\rangle / J^{2}$ a alors pour expression :

$$
\frac{\langle c\rangle}{J^{2}}=\frac{\frac{P}{4 \pi} \cdot \int_{\theta_{1}}^{\theta_{2}} j^{2} \cdot \frac{Z_{2}-Z_{1}}{\theta_{2}-\theta_{1}} \cdot \mathrm{d} \theta}{\frac{1}{2 \pi} \cdot \int_{\theta_{1}}^{\theta_{2}} j^{2} \cdot \mathrm{d} \theta}=\frac{P \cdot\left(Z_{2}-Z_{1}\right)}{2 \cdot\left(\theta_{2}-\theta_{1}\right)} .
$$

On notera que si $z$ varie encore à l'extérieur de l'intervalle $] \theta_{1}, \theta_{2}$ [, cette variation n'est pas utilisée dans le cadre de l'optimisation choisie. Si l'on suppose que $z\left(\theta_{1}\right)=Z_{0}$ et $z\left(\theta_{2}\right)=Z_{M}$ (variation trapézoïdale de $z$ ) et que $\Delta Z=Z_{M}-Z_{0}$ reste constant, on peut remarquer que $\langle c\rangle / J^{2}$ (inversement proportionnel

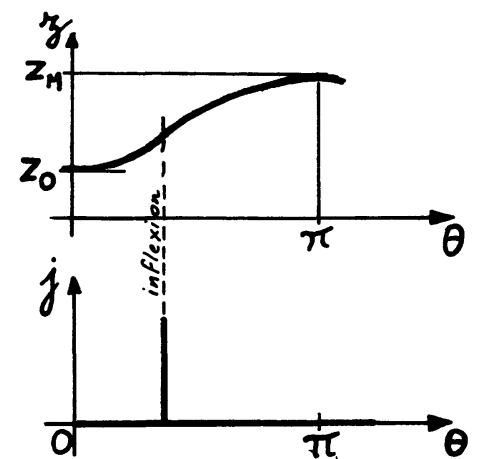

a)

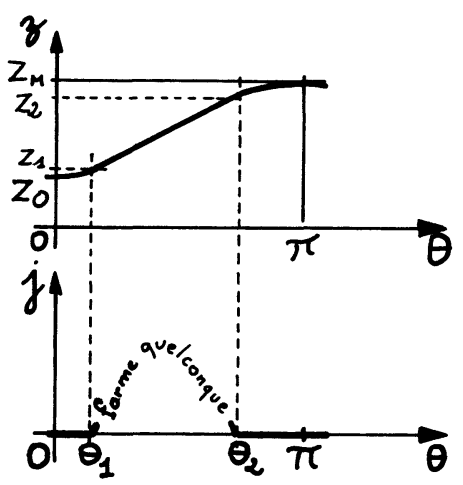

b)

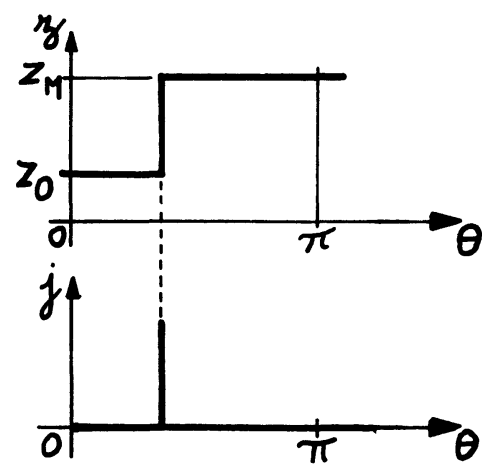

c)

Fig. 3. - Formes de courant maximisant $\langle c\rangle / J^{2}$ en régime linéaire pour une perméance présentant : a) un point d'inflexion; b) une partie à croissance constante; c) un saut.

[Current shape maximizing $\langle c\rangle / J^{2}$ in linear domain for a permeance with : a) an inflection point; b) a constant increase part; c) a leap.]

à $\theta_{2}-\theta_{1}$ ) sera maximal pour $\theta_{2}-\theta_{1}=0$, ce qui correspond à un courant en forme d'impulsion de Dirac synchronisé sur le saut de perméance (Fig. 3c).

3. 3 CONCLUSIONS SUR LES FORMES DE COURANT OPTIMALES EN RÉGIME NON SATURÉ. - L'étude précédente montre la grande diversité des formes d'ondes de courant en fonction du critère d'optimisation choisi 
et de la forme de perméance de la machine. Par exemple, si la variation de la perméance est une fonction sinusoïdale de l'angle et si l'on se donne un nombre maximal d'ampères-tours, c'est la forme rectangulaire du courant qui donne le couple moyen le plus élevé. $\mathrm{Si}$, par contre, on veut maximiser le couple moyen à ampères-tours efficaces donnés, c'est alors un courant en impulsion de Dirac qui convient le mieux. Dans le premier cas, le convertisseur fournit de l'énergie pendant un temps maximal (une demi- période). Dans le second cas, il conduit un temps minimal.

En fait, les formes d'ondes ainsi déterminées sont toutes théoriques et pas nécessairement physiquement réalisables. Elles permettent essentiellement de connaître le maximum de couple qu'il est possible d'obtenir dans le meilleur des cas et par conséquent l'écart de performances entre une alimentation idéale et une alimentation réelle. C'est ce que donnent le tableau I pour le critère $\langle c\rangle / J_{\mathrm{M}}^{2}$ et le tableau II pour le critère

Tableau I. - Comparaison des performances obtenues par diverses formes d'ondes de courant par rapport aux formes d'ondes optimales (critère $\langle c\rangle / J_{\mathrm{M}}^{2}$ ).

[Comparison of the performance obtained by various curent waveforms with reference to optimal waveforms (criterion $\left.\left.\langle c\rangle / J_{\mathrm{M}}^{2}\right) \cdot\right]$

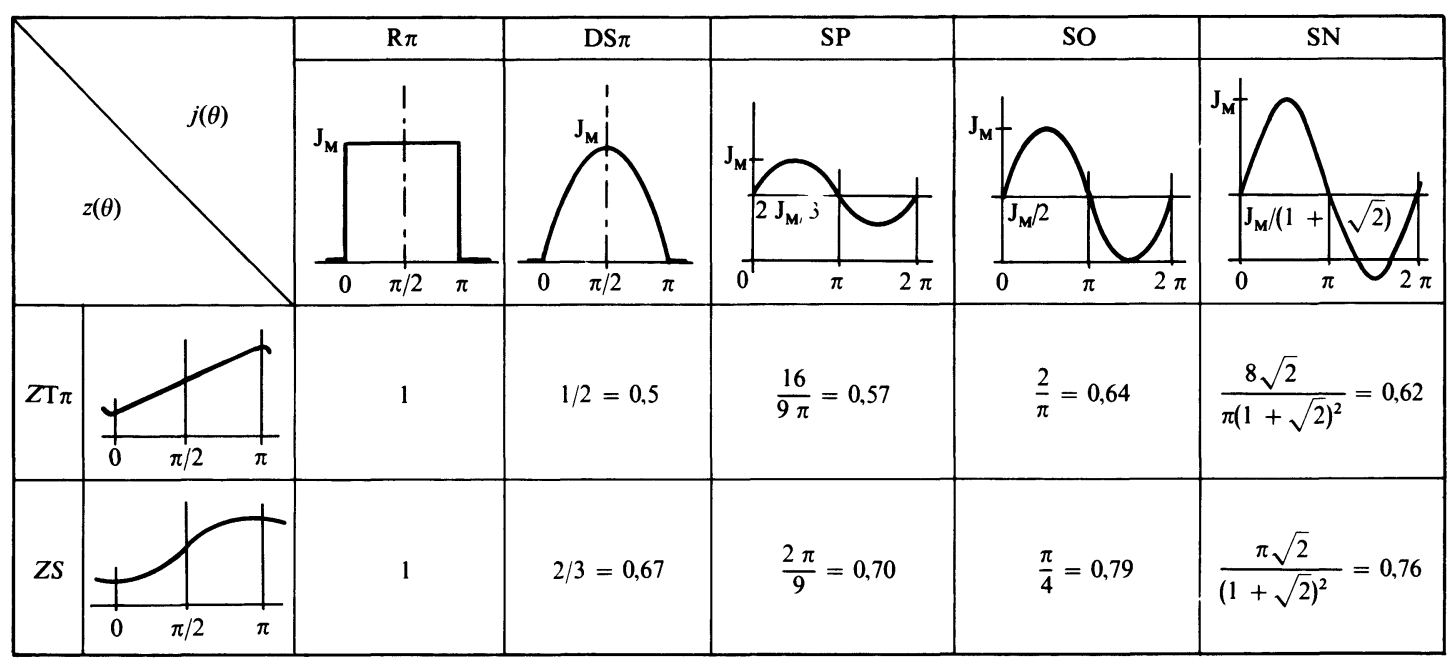

Tableau II. - Comparaison des performances obtenues par diverses formes d'ondes de courant par rapport aux formes d'ondes optimales (critère $\langle c\rangle / J^{2}$ ).

[Comparison of the performance obtained by various current waveforms with reference to optimal waveforms $\left(\right.$ criterion $\left.\langle c\rangle / J^{2}\right)$.]

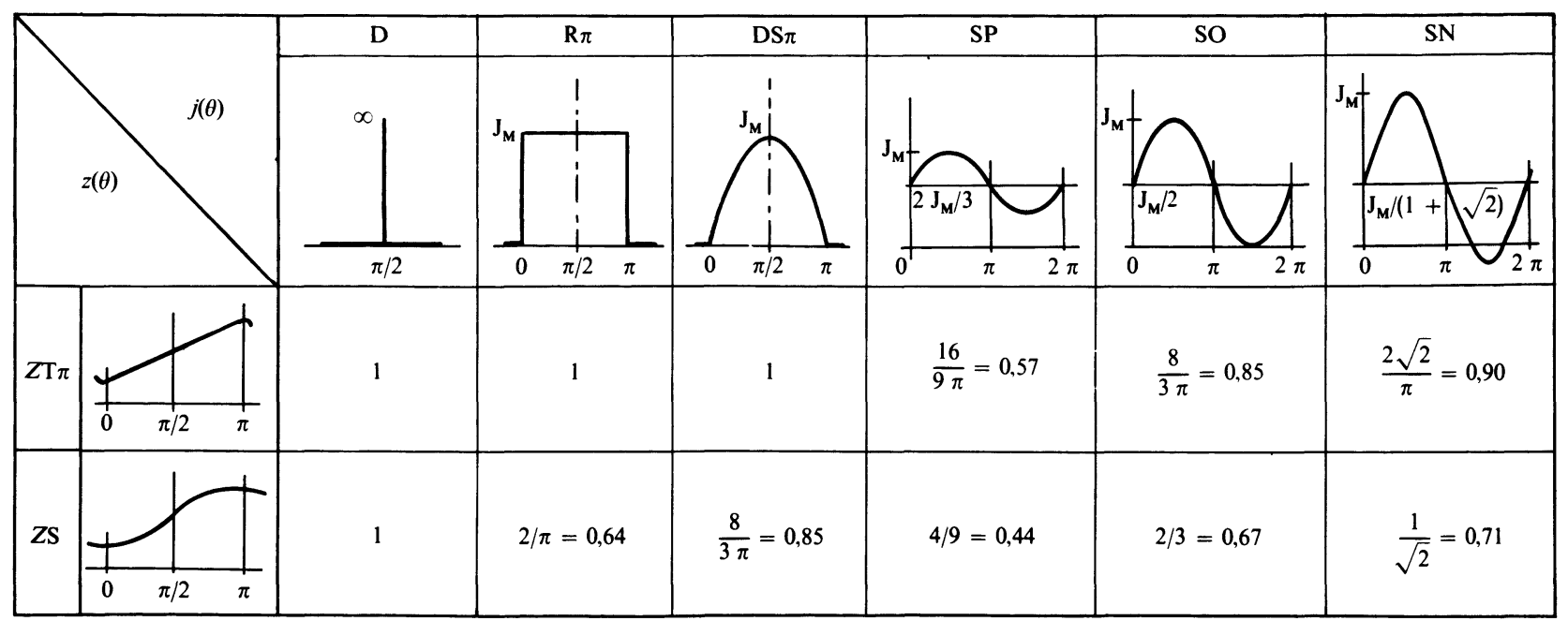


$\langle c\rangle / J^{2}$ pour deux allures de perméance typiques : triangulaire $(Z \mathrm{~T} \pi)$ et sinusoïdale $(Z S)$.

Avec une perméance triangulaire, la perte de performance est sensible dès que l'on s'écarte de la forme d'onde rectangulaire. Avec une onde de courant semisinusoïdale, la puissance convertible n'est que la moitié de ce qu'elle est avec une onde rectangulaire. Avec une onde de courant sinusoïdale superposée à une composante continue, le maximum du rapport $\langle c\rangle / J_{M}^{2}$ est obtenu pour une composante continue égale à la valeur crête de la sinusoïde (SO). Il ne vaut que $64 \%$ de la valeur théorique maximale. De plus, le couple instantané présente des valeurs négatives car il y a du courant dans la zone où $\mathrm{d} z / \mathrm{d} \theta$ est négative. Ces valeurs négatives de couple engendrent des vibrations et nuisent à la résistance mécanique des machines monophasées.

Avec une perméance sinusoïdale (ZS), les pertes de performances sont moins importantes si l'on centre le maximum du courant sur le point d'inflexion de la perméance $(\theta=\pi / 2)$ mais les conclusions générales restent les mêmes.

Pour le critère $\langle c\rangle / J^{2}$ et une perméance trapézoïdale, nous avons montré que la forme d'onde n'importait pas si le courant était nul dans la partie décroissante de $z(\theta)$ soit $\mathrm{d} z / \mathrm{d} \theta<0$. Cest ce que confirme le tableau II. L'utilisation d'un courant sinusoïdal superposé à une composante continue, bien que créant un couple instantané négatif, peut être envisagé avec une perte de performance très raisonnable $(10 \%)$ si la valeur crête de la sinusoïde est égale à $\sqrt{2}$ fois la valeur moyenne.

Toujours pour le critère $\langle c\rangle / J^{2}$ et avec une perméance sinusoïdale, la présence du point d'inflexion pénalise les formes d'ondes trop éloignées de l'impulsion de Dirac. En choisissant une onde rectangulaire d'ouverture $\Delta \theta$ centrée sur le point d'inflexion, le rapport $\langle c\rangle / J^{2}$ normalisé (Tableau III) a pour valeur $\sin (\Delta \theta / 2) /(\Delta \theta / 2)$ qui décroît de 1 à 0,64 lorsque
$\Delta \theta$ varie de 0 à $\pi$. Avec une onde semi-sinusoïdale d'ouverture $\mathrm{D} \theta$ centrée sur le point d'inflexion, le rapport $\langle c\rangle / J^{2}$ a pour valeur :

$$
\frac{\sin (\Delta \theta / 2)}{\Delta \theta / 2} \cdot \frac{1}{1-\left(\frac{\Delta \theta}{2 \pi}\right)^{2}}>\frac{\sin (\Delta \theta / 2)}{\Delta \theta / 2}
$$

pour

$$
0<\Delta \theta<2 \pi .
$$

Le choix d'une forme d'onde demi-sinusoïdale d'ouverture 1,77 rd $\left(101^{\circ}\right)$ ne provoque alors que $5 \%$ de chute de performance par rapport au maximum théorique.

Enfin, on peut remarquer que les machines dont les formes de perméance sont triangulaires ou trapézoïdales présentent l'avantage de satisfaire aux deux critères d'optimisation (Fig. 4). De plus, l'association mécanique d'au moins deux machines alimentées en polyphasé permet d'obtenir une ondulation de couple nulle sur l'arbre de sortie (Fig. 5). Notons aussi que pour ces formes d'ondes, $\mathrm{d} z / \mathrm{d} \theta$ et $j$ sont constants dans l'intervalle $2 \pi / q$. La tension aux bornes de l'enroulement $(l . \mathrm{d} i / \mathrm{d} t+i . \mathrm{d} l / \mathrm{d} t+R . i)$ est constante dans l'intervalle considéré. Hors commutations, le couple constant peut alors être obtenu aussi bien avec une alimentation en créneau de tension que de courant. Il apparaît ainsi l'intérêt de concevoir une machine en fonction de son alimentation par convertisseur statique afin de satisfaire à plusieurs critères d'optimisation qui, en règle générale, aboutissent à des solutions incompatibles.

\section{Optimisation en régime saturé.}

Les machines électrotechniques fonctionnent la plupart du temps en partie en saturation. Il est donc nécessaire d'examiner comment se comportent alors les machines que nous étudions. Nous allons chercher

Tableau III. - Comparaison des performances obtenues par diverses formes d'ondes de courant par rapport aux formes d'ondes optimales (critère $\left.\langle c\rangle / J^{2}\right)$.

[Comparison of the performance obtained by various current waveforms with reference to optimal waveforms (criterion $\left.\langle c\rangle / J^{2}\right)$.]

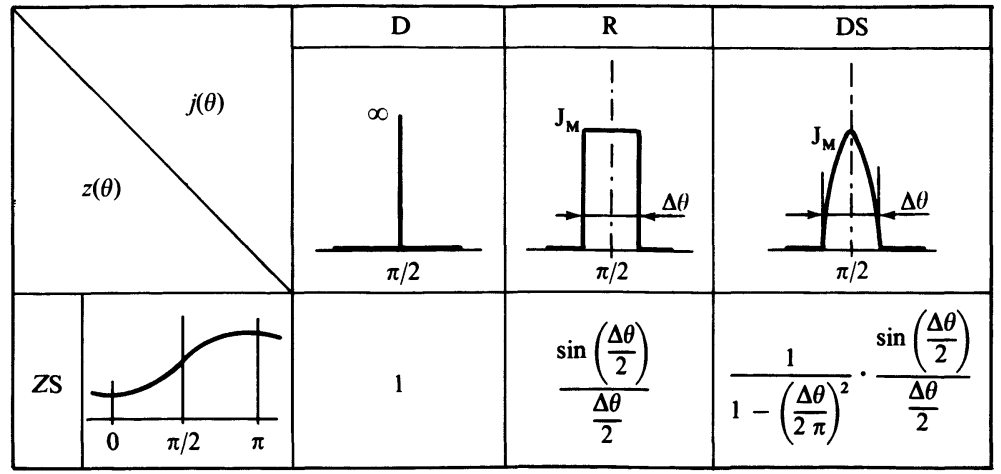




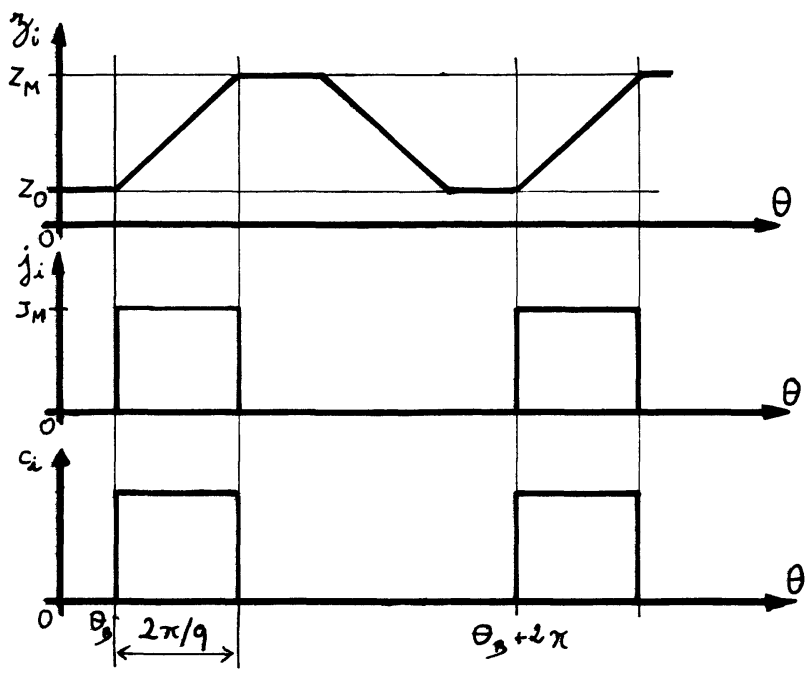

Fig. 4. - Formes de perméance et de courant maximisant les deux rapports $\langle c\rangle / J_{\mathrm{M}}^{2}$ et $\langle c\rangle / J^{2}$.

[Forms of permeance and current maximizing the two ratios $\langle c\rangle / J_{M}^{2}$ and $\langle c\rangle / J^{2}$.]
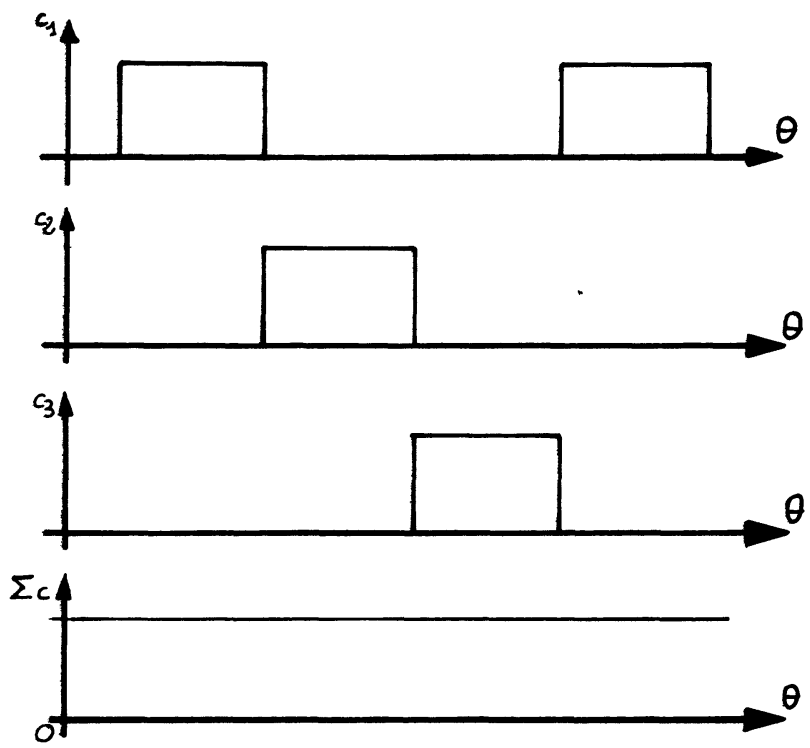

Fig. 5. - Couples élémentaire et total pour une machine $q$-phasée (ici $q=3$ ).

[Torques for 1-phase and $q$-phase machines (here $q=3$ ).]

les formes optimales de courant suivant les deux critères d'optimisation déjà exposés. Cherchant bien évidemment des résultats applicables à n'importe quel type de machine à réluctance variable, il nous faut choisir un modèle qui représente assez bien toute cette classe de machines. Le modèle à trois pentes (Fig. 6) est très proche de la réalité pour des machines convenablement construites $[9,11]$. A l'opposition, la relation $\phi(j)$ est linéaire en deçà et au-delà de $J_{\mathrm{s}}$. Le rapport $\phi / j$ (perméance) est toujours noté $Z_{0}$. A la conjonction, la perméance est maximale pour des

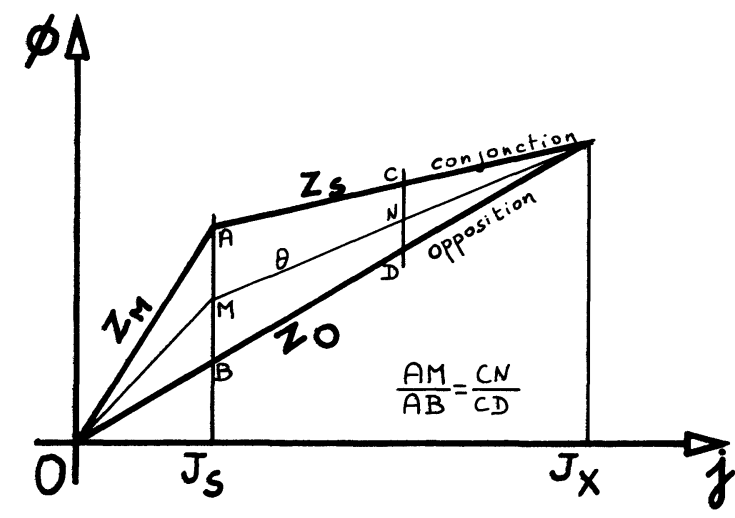

Fig. 6. - Modèle de la machine pour la simulation.

[Equivalent model of the machine for simulation.]

ampères-tours inférieurs à $J_{\mathrm{S}}$, et est encore notée $Z_{\mathrm{M}}$. Au-delà de $J_{\mathrm{S}}$ (domaine de saturation), $\partial \phi / \partial j=Z_{\mathrm{S}}$. On a de plus les relations suivantes:

$$
\begin{aligned}
& Z_{\mathrm{M}}>Z_{0}>0 \\
& Z_{\mathrm{M}}>Z_{\mathrm{S}}>0
\end{aligned}
$$

et en général :

$$
Z_{0}>Z_{\mathrm{s}} \text {. }
$$

Nous noterons $\Delta Z$ la différence $Z_{\mathrm{M}}-Z_{0}$ (différence maximale des perméances en linéaire). Pour normaliser la saturation, nous introduisons le paramètre $K$ :

$$
Z_{\mathrm{S}}=K \cdot Z_{\mathrm{M}}+(1-K) \cdot Z_{0}
$$

soit :

$$
K=\frac{Z_{\mathrm{S}}-Z_{0}}{Z_{\mathrm{M}}-Z_{0}} .
$$

$K$ est le rapport de la différence des pentes en saturation sur la différence des pentes en linéaire. Une valeur de $K$ négative correspond au modèle le plus général des machines à réluctance variable. En saturation, les droites se rejoignent pour un courant noté $J_{\mathbf{x}}$. Au-delà, elles sont confondues. $J_{\mathbf{x}}$ a pour expression :

$$
J_{\mathrm{X}}=\frac{Z_{\mathrm{M}}-Z_{\mathrm{S}}}{Z_{\mathrm{0}}-Z_{\mathrm{S}}}=\frac{1-K}{-K} \cdot J_{\mathrm{s}}
$$

Entre les positions d'opposition et de conjonction, le rapport $\phi / j$ est donné par une interpolation des deux courbes extrêmes suivant une fonction choisie pour représenter au mieux la machine à étudier.

4.1 MAXIMISATION DU RAPPORT $\langle c\rangle / J_{\mathrm{M}}^{2}$. - La maximisation du rapport $\langle c\rangle / J_{\mathrm{M}}^{2}$ revient à maximiser le rapport de l'aire $A$ délimitée par la courbe décrite par le point de fonctionnement dans le plan $\phi(j)$. La relation entre couple moyen $\langle c\rangle$ et aire $A$ est :

$$
\langle c\rangle=\frac{P \cdot A}{2 \pi} \text {. }
$$


La maximisation de cette surface est évidente : il faut remplir le plus possible le cycle $\phi(j)$ quand la machine passe de l'opposition à la conjonction (Fig. 7).

La forme du courant optimale est donc rectangulaire. Les rectangles successifs peuvent être unidirectionnels (onde à valeur moyenne non nulle) ou alternés (onde alternative pure). Le couple moyen obtenu a pour valeur :

$$
\begin{aligned}
& \langle c\rangle=\frac{P}{4 \pi} \cdot \Delta Z \times \\
& \times J_{S}^{2}\left[K\left(\frac{J_{M}}{J_{S}}\right)^{2}+2(1-K)\left(\frac{J_{M}}{J_{S}}\right)+K-1\right] .
\end{aligned}
$$

On peut noter que la forme d'onde optimale et le couple moyen obtenu ne dépendent pas de la variation de la perméance en fonction de l'angle.

4.2 Maximisation du RapPort $\langle c\rangle / J^{2}$. - Contrairement au cas non saturé, la réponse à la question posée n'est pas unique et dépend du degré de saturation auquel est utilisée la machine. On doit tenir compte de la valeur de la perméance en saturation $Z_{\mathrm{s}}$. Il est donc indispensable de donner les résultats sous forme de courbes. Nous représentons le rapport recherché $\left(\langle c\rangle / J^{2}\right)$ en fonction des ampères-tours normalisés par rapport au courant de saturation $J_{S}$ et avec $K$ comme paramètre. Nous déterminons ces courbes pour deux lois de variation de la perméance en fonction de $\theta$ : sinusoïdale et trapézoïdale.

4.2.1 Perméance sinusoídale. - Pour connaître la forme exacte des courbes, il est nécessaire de faire un calcul s'appuyant sur le modèle de variation choisi. Dans cette étude, à $j$ donné, $\phi$ s'écrit :

$$
\phi(\theta)=\frac{\phi(0)+\phi(\pi)}{2}-\frac{\phi(\pi)-\phi(0)}{2} \cdot \cos \theta .
$$

Pour $j \leqslant J_{\mathrm{s}}$ :

$$
z_{j<J_{\mathbf{s}}}=\frac{Z_{M}+Z_{0}}{2}-\frac{\Delta Z}{2} \cdot \cos \theta .
$$

Pour $j \geqslant J_{\mathbf{s}}$ :

$$
\begin{aligned}
z_{j>J_{\mathrm{s}}}=\frac{Z_{\mathrm{s}}+Z_{0}}{2}- & \frac{Z_{\mathrm{S}}-Z_{0}}{2} \times \\
& \times \cos \theta+\frac{\phi_{\mathrm{s}}}{2 j} \cdot(1-\cos \theta)
\end{aligned}
$$

avec

$$
\phi_{\mathrm{S}}=\left(Z_{\mathrm{M}}-Z_{\mathrm{S}}\right) \cdot J_{\mathrm{S}}=(1-K) \cdot \Delta Z . J_{\mathrm{S}} .
$$

Le couple électromagnétique produit par une machine à réluctance variable peut s'écrire [12] :

$$
c=-\int_{0}^{j} \frac{\partial z\left(j^{\prime}, \theta_{\mathrm{m}}\right)}{\partial \theta_{\mathrm{m}}} \cdot j^{\prime} \cdot \mathrm{d} j^{\prime}
$$

où $\theta_{\mathrm{m}}=\theta / P$. On peut en déduire le couple en régime

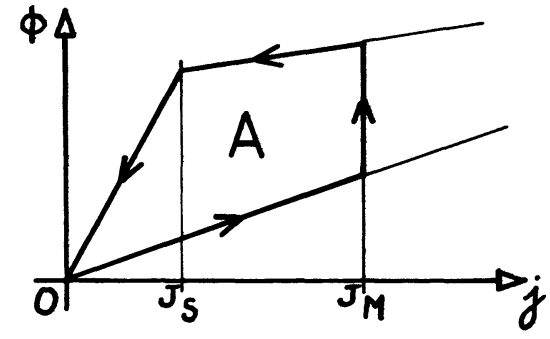

Fig. 7. - Maximisation du rapport $\langle c\rangle / J_{\mathrm{M}}^{2}$ en saturé. [Maximization of $\langle c\rangle / J_{M}^{2}$ in saturated domain.]

de saturation :

$$
c=\int_{0}^{J_{\mathbf{s}}} \frac{\mathrm{d} z_{j<J_{\mathbf{s}}}}{\partial \theta / P} \cdot j^{\prime} \cdot \mathrm{d} j^{\prime}+\int_{J_{\mathbf{s}}}^{j} \frac{\mathrm{d} z_{j>J_{\mathbf{s}}}}{\partial \theta / P} \cdot j^{\prime} \cdot \mathrm{d} j^{\prime}
$$

soit tous calculs effectués :

$$
\begin{aligned}
c_{j>J_{\mathbf{s}}} & =\frac{1}{2} \cdot \sin \theta \times \\
\times & {\left[\frac{\Delta Z}{2} \cdot J_{\mathbf{S}}^{2}+\frac{K \cdot \Delta Z}{2} \cdot\left(j^{2}-J_{\mathbf{S}}^{2}\right)+\phi_{\mathrm{S}} \cdot\left(j-J_{\mathrm{S}}\right)\right] }
\end{aligned}
$$

qui se simplifie pour la partie linéaire en :

$$
c_{j<J_{\mathrm{s}}}=\frac{1}{2} \cdot \sin \theta \cdot \frac{\Delta Z}{2} \cdot j^{2} .
$$

Ces expressions sont trop compliquées pour pouvoir effectuer analytiquement la maximisation de $\langle c\rangle / J^{2}$. Aussi est-il nécessaire d'utiliser une méthode d'optimisation numérique. A cause des temps de calculs prohibitifs des programmes traditionnels, nous avons été amené à mettre au point un programme d'optimisation plus spécialisé et, par conséquent, beaucoup plus rapide (voir annexe I). Les résultats de cette optimisation sont représentés sur la figure 8 . Le rapport $\langle c\rangle / J^{2}$, multiplié par $4 / P . \Delta Z$ à fin de normalisation, est donné en fonction du rapport des ampères-tours efficaces $J$ aux ampères-tours de saturation $J_{\mathrm{S}}$ du modèle de la figure 6 .

Pour des ampères-tours faibles $\left(J / J_{\mathrm{S}} \ll 1\right)$, toutes les machines donnent des résultats identiques (à $P$ et $\Delta Z$ donnés) aux machines linéaires représentées par la droite de paramètre $K=1$. Les performances diminuent ensuite quand $K$ diminue (saturation de plus en plus marquée) et quand $J / J_{\mathrm{S}}$ augmente. Pour $K$ négatif, on remarque l'hyperbole de limitation pratique (trait + hachures) correspondant à un remplissage total du cycle (courant rectangulaire).

Toutes ces courbes montrent la puissance maximale que l'on peut tirer d'une machine (paramètre $K$ ) pour des pertes Joule données (abscisse $J / J_{\mathbf{S}}$ ). On indique en particulier cette puissance maximale pour des ampères-tours crêtes égaux à $2 . J_{\mathbf{S}}$. 


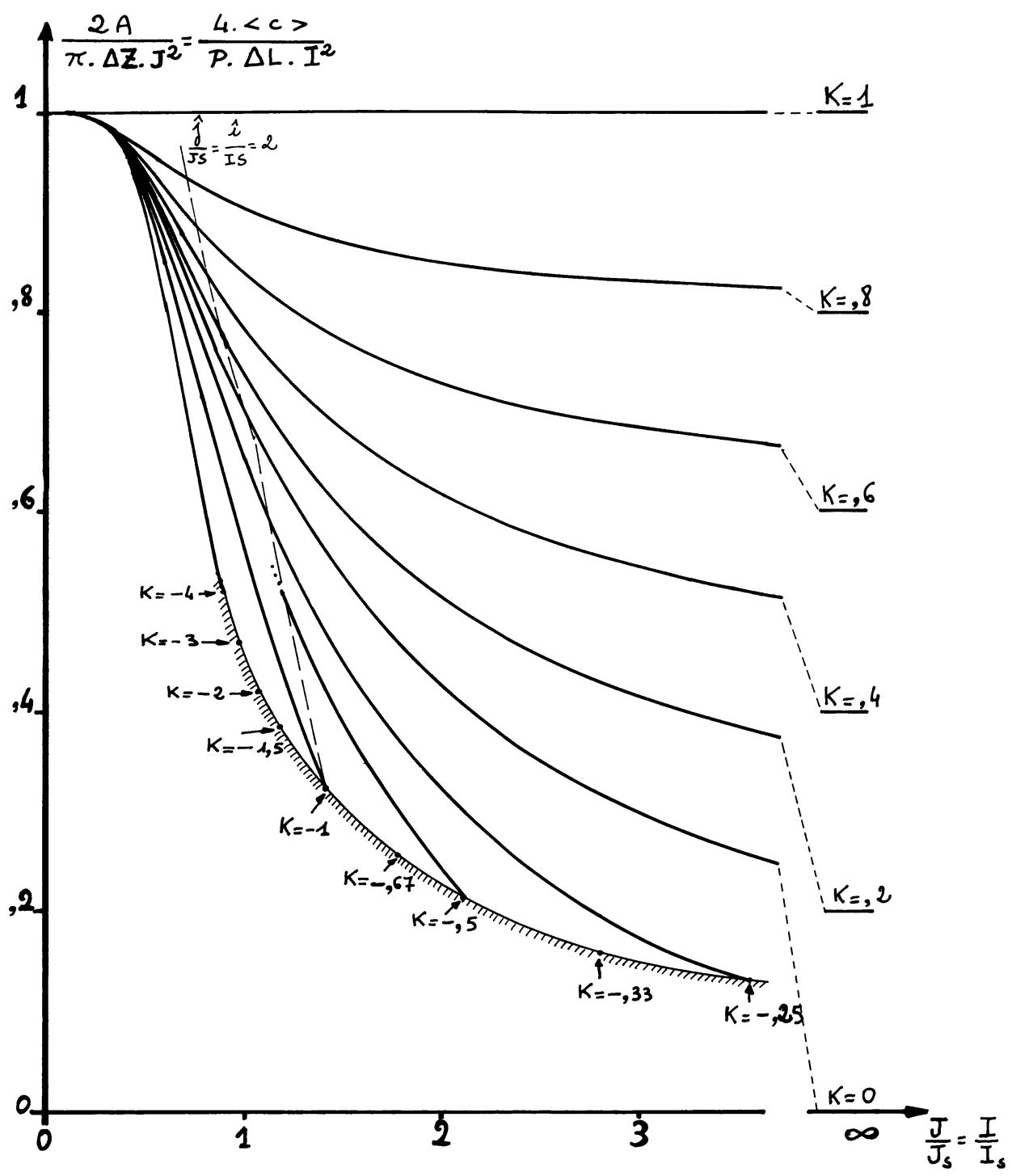

Fig. 8. - Rapport $\langle c\rangle / J^{2}$ normalisé fonction de $K$ et du courant efficace normalisé dans une machine à variation de perméance sinusoïdale.

[Normalized ratio $\langle c\rangle / J^{2}$ as a function of $K$ and of the RMS curent in a sine permeance variation machine.]

Le programme décrit précédemment fournit aussi les formes de courant optimales pour chaque point de la courbe précédente. En linéaire $(K=1)$, on retrouve évidemment le Dirac de courant qui donne un Dirac de couple dont le développement en série de Fourier est un spectre où tous les harmoniques sont présents et d'amplitudes égales. A l'opposé, pour $J=J_{\mathbf{x}} / \sqrt{2}$, le courant est nécessairement rectangulaire (remplissage de tout le cycle $\phi(j)$ ). Le courant et le couple instantanés ainsi que le développement en série de Fourier sont représentés sur la figure 9 pour $J / J_{S}=1$.

Des formes d'ondes pour des valeurs de $K$ intermédiaires sont données figures 10 et 11 . Entre ces valeurs de $K$, les formes d'ondes varient progressivement. De 0 à $\pi / 2$, ces formes d'ondes sont constituées d'un palier à courant nul, puis d'un front d'amplitude $J_{\mathrm{S}}$, puis d'un motif variable suivant $K$. Elles sont symétriques par rapport à $\pi / 2$ jusqu'à $\pi$ et nulles ensuite jusqu'à $2 \pi$.

Ces résultats permettent aussi de choisir le nombre de phases intéressant pour l'alimentation de ce type de machine. On peut montrer que dans une machine $q$-phasée, seuls les harmoniques de rang multiple de $q$ s'ajoutent (car ils sont en phase) et que tous les autres s'annulent. L'alimentation par créneau de courant impose alors une machine à nombre de phases impair et supérieur ou égal à 3 (Fig. 9). Il ne subsiste alors sur l'arbre que le couple moyen sans ondulation décelable. Ce raisonnement est encore valable pour $K=0$ et $K=1$ (Fig. 10), mais avec $q$ impair, supérieur ou égal à 5 . On peut éventuellement faire le choix de $q=4$ pour éliminer l'harmonique 3 sans trop augmenter le nombre de phases. Pour des formes de courant de plus en plus proches du Dirac, la machine $q$-phasée élimine les harmoniques jusqu'au rang $q-1$. 


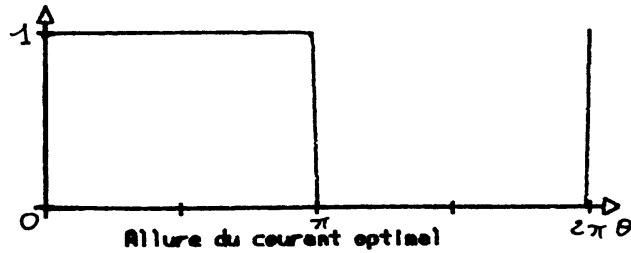

$$
\begin{gathered}
K=-2,41297 \\
J / J_{\mathbf{S}}=1
\end{gathered}
$$
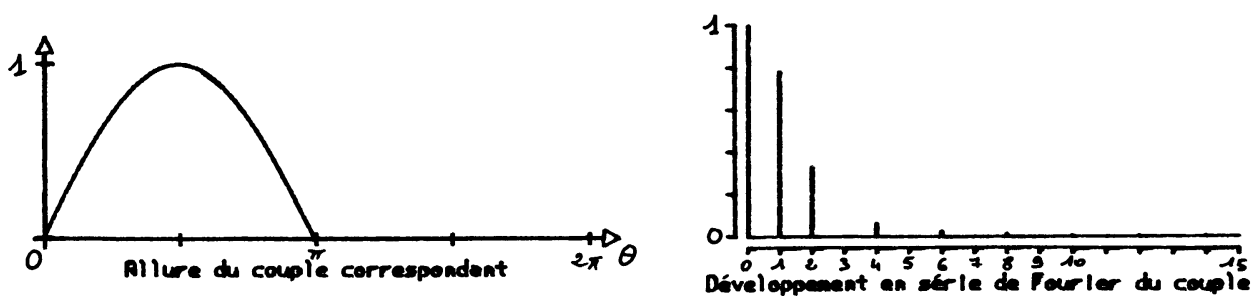

Fig. 9.

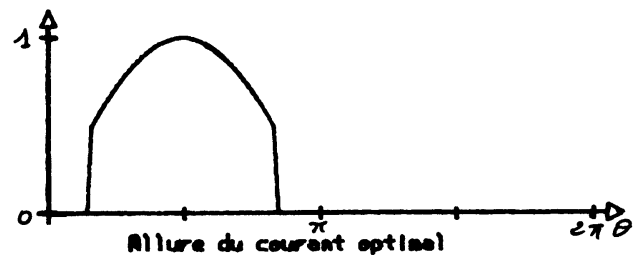

$$
\begin{gathered}
K=0 \\
J / J_{\mathrm{S}}=1
\end{gathered}
$$
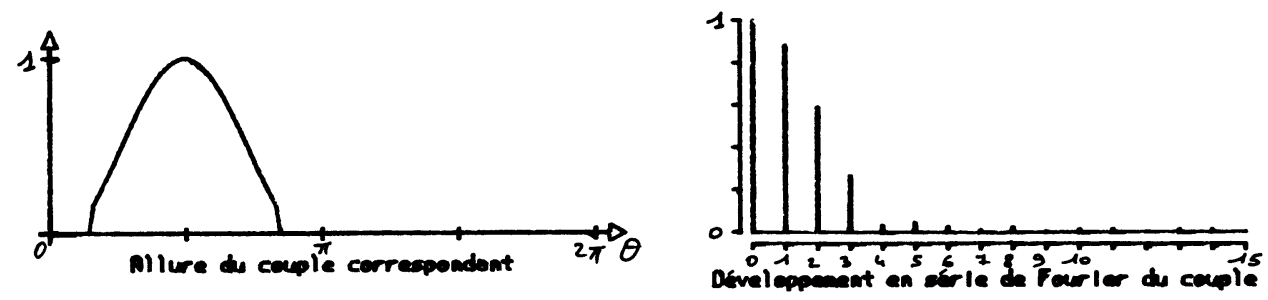

Fig. 10.

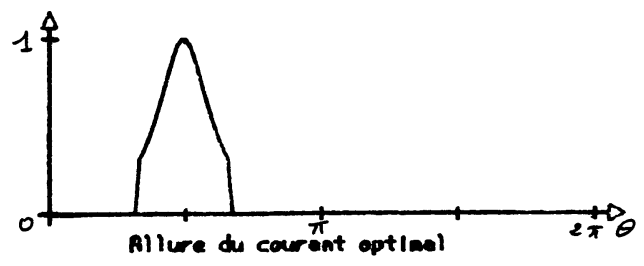

$$
\begin{aligned}
& K=0,8 \\
& J / J_{\mathbf{S}}=1
\end{aligned}
$$
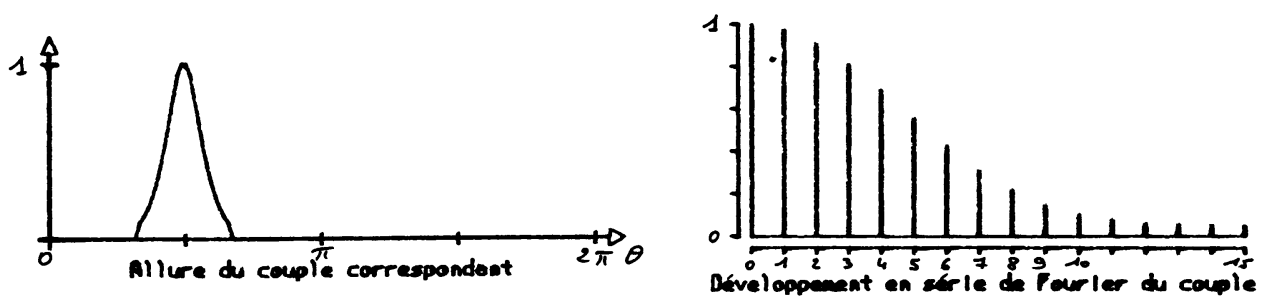

Fig. 11. 
Le fonctionnement dans ou près du domaine linéaire, s'il permet de sortir plus de couple de la machine à pertes Joule données, n'est pas très satisfaisant ni pour la charge qui voit un couple pulsatoire très important, ni pour le convertisseur d'alimentation qui doit contrôler de fortes tensions pour faire croître et décroître rapidement le courant.

4.2.2 Perméance trapézoïdale. - On choisit maintenant le modèle de perméance trapézoïdale dessiné figure 12 pour la partie linéaire complété pour la saturation par le modèle à trois pentes de la figure 6 . On conserve la définition de $K$ donnée par les relations (9) et (10).

Entre 0 et $\Delta \theta$, la perméance s'écrit :

- en linéaire :

$$
Z_{0}+\frac{\Delta Z}{\Delta \theta} \cdot \theta
$$

- en saturé :

$$
\begin{aligned}
\left(Z_{0}+\frac{\Delta Z}{\Delta \theta} \cdot \theta\right) \cdot \frac{J_{s}}{j}+\left(Z_{0}+K \cdot \frac{\Delta Z}{\Delta \theta} \cdot \theta\right) & \times \\
& \times\left(1-\frac{J_{s}}{j}\right)
\end{aligned}
$$

et le couple instantané :

- en linéaire :

$$
\frac{P}{2} \cdot \frac{\Delta Z}{\Delta \theta} \cdot j^{2}
$$

- en saturé :

$$
\frac{P}{2} \cdot \frac{\Delta Z}{\Delta \theta} \cdot\left[\left(\frac{2 j}{J_{s}}-1\right)+K\left(\frac{j}{J_{s}}-1\right)^{2}\right] \cdot J_{\mathrm{S}}^{2}
$$

La non-dépendance en $\theta$ des expressions du coūple permet d'effectuer une détermination analytique de la forme du courant maximisant le rapport couple moyen sur carré du courant efficace. On en déduit aisément que la forme d'onde optimale est rectangulaire telle que $j=J \sqrt{2 \pi / \Delta \theta}$ pour $0 \leqslant \theta \leqslant \Delta \theta$ et $j=0$ partout ailleurs. Le couple moyen résultant est alors :

$$
\langle c\rangle=\frac{P}{2} \cdot \frac{\Delta Z}{\Delta \theta} \cdot J^{2} .
$$

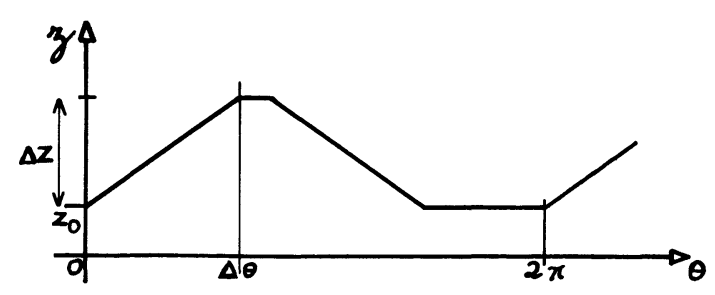

Fig. 12. - Modèle de perméance trapézoïdale.

[Model of trapezoidal permeance.]
En linéaire, nous avons déjà vu que la forme d'onde était indifférente, le couple moyen valant alors :

$$
\frac{P}{2} \cdot \frac{\Delta Z}{\Delta \theta} \cdot J^{2}
$$

jusqu'à $J=J_{\mathrm{s}} \sqrt{\Delta \theta / 2 \pi}$.

Il est intéressant de comparer les résultats obtenus avec une machine à variation de perméance sinusoïdale par rapport à ceux obtenus avec une machine à perméance trapézoïdale. Dans ce dernier cas, les effets de la saturation n'apparaissent que pour les ampèrestours supérieurs à $J_{\mathrm{s}} \sqrt{\Delta \theta / 2 \pi}$ ce qui implique un rapport $\langle c\rangle / J^{2}$ plus favorable à la machine à perméance trapézoïdale (rappelons que la forme d'onde optimale de courant dans une machine à variation de perméance sinusoïdale fait toujours intervenir la saturation).

D'autre part, si $\Delta \theta$ est inférieur à $2 \operatorname{rad}\left(114^{\circ}\right)$, à courant efficace donné, la machine à perméance trapézoïdale donne un couple moyen plus important qu'une machine à variation de perméance sinusoïdale. Ce qui montre ici encore l'intérêt des machines à perméance trapézoïdale dans le cadre de machines $q$-phasées (avec $q>3$ ), ou tout au moins des machines à taux de variation constant et présentant une variation utile sur un faible angle électrique. Le faible rapport cyclique de fonctionnement de telles machines doit être compensé par une augmentation du nombre de phases pour limiter l'ondulation résultante de couple.

\section{Machines excitées.}

Dans les machines que nous avons étudiées jusqu'ici, les ampères-tours étaient créés par une seule bobine, le courant y circulant pouvant être à valeur moyenne nulle ou non. Une telle machine est dite « à un enroulement " (ou encore " non excitée »). Dans le cas où les ampères-tours présentent une composante continue, il est possible d'injecter tout ou partie de celle-ci dans un premier bobinage. Un second bobinage est alors spécialisé dans la création des ampères-tours restants (essentiellement variables). Ce type de machine est dit « à deux enroulements » (ou encore " excité »). La distinction entre les deux bobines est justifiée par le fait que le coût de production des ampères-tours continus est souvent moindre que celui des ampères-tours variables. En effet, un convertisseur délivrant un courant continu est en général plus simple, de dimensionnement plus faible et de rendement meilleur qu'un convertisseur délivrant un courant variable de même valeur efficace. Il est même parfois possible de se dispenser de ce convertisseur comme dans le montage de la figure 13 où le courant continu de la boucle entre redresseur et onduleur est utilisé pour l'alimentation de la bobine d'excitation. Dans une telle configuration, le coût d'exploitation de la bobine " continue " est dû essentiellement aux pertes Joule dans cette bobine alors que pour les bobines alternatives, il faut prendre en 


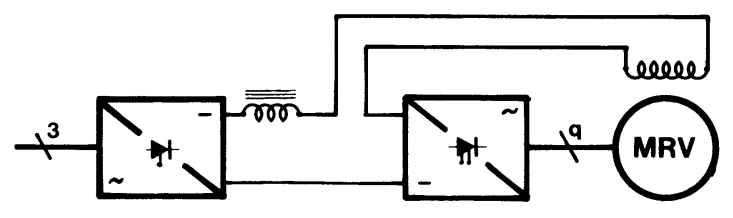

Fig. 13. - Excitation sans convertisseur auxiliaire d'une machine à réluctance variable.

[Excitation without an auxiliary converter of a reluctance machine (R.M.).]

compte, en plus des pertes Joule propres, les pertes dans les convertisseurs. Mieux encore, l'utilisation d'aimants permanents pour la fourniture des ampèrestours continus donne un coût d'exploitation nul. Dans tous les cas, les ampères-tours continus peuvent être considérés comme une polarisation dans le plan $\phi(j)$ qui amène le point de fonctionnement dans une zone plus intéressante.

Les considérations précédentes montrent l'intérêt de reprendre les calculs d'optimisation quand le coût des ampères-tours de polarisation est différent de celui des ampères-tours variables. Nous supposons désormais que les ampères-tours continus représentent la valeur moyenne $\langle j\rangle$ des ampères-tours optimaux et que les ampères-tours variables sont la partie purement alternative $J_{\mathrm{A}}$ de ce même signal optimal. La fonction à optimiser est maintenant :

$$
\frac{\langle c\rangle}{J^{\prime 2}}=\frac{\langle c\rangle}{J_{A}^{2}+\alpha_{\cdot}\langle j\rangle^{2}}
$$

où $\alpha$ représente le coefficient de pondération du carré des ampères-tours moyens par rapport au carré des ampères-tours alternatifs.

5.1 MAXIMISATION DU RAPPORT $\langle c\rangle / J^{\prime 2}$ POUR UNE PERMÉANCE SINUSOÏDALE. - Par une méthode numérique dérivée de celle utilisée pour les machines à un enroulement (voir annexe I), on peut trouver la forme d'onde d'alimentation optimale et le rapport $\langle c\rangle / J^{\prime 2}$ correspondant à une machine donnée et à $\alpha$ donné. Le premier résultat intéressant est le gain obtenu sur le couple. On représente figure 14 le couple moyen pour $\alpha$ compris entre 0 et 1 par rapport au couple moyen obtenu pour $\alpha=1$.

On peut immédiatement constater que le gain n'est vraiment sensible que pour $\alpha$ inférieur à 0,4 et est infini pour $\alpha$ nul. Pour $\alpha=0,2$, il vaut $67 \%$. D'autre part, on constate que, à $J^{\prime} / J_{\mathrm{S}}$ et $K$ donnés, le rapport $\langle c\rangle / J^{\prime 2}$ pour $\alpha<1$ est toujours supérieur à $\langle c\rangle / J^{2}$ pour $\alpha=1$. Cela montre l'intérêt des machines à réluctance variable excitées et ce, d'autant plus que les ampères-tours d'excitations sont moins "coûteux " à produire que les ampères-tours alternatifs.

La figure 15 représente diverses allures de courant optimales pour $J^{\prime}=1$ dans diverses machines $(K$ variable). .On y remarque que les ampères-tours ne sont maintenant plus nuls pour $\pi \leqslant \theta \leqslant 2 \pi$. Le couple

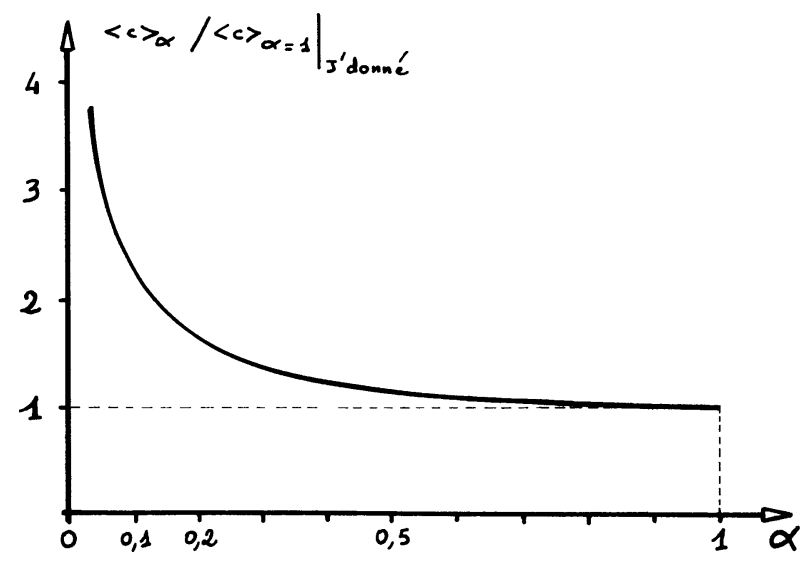

Fig. 14. - Gain de couple en fonction du paramètre $\alpha$. [Torque gain with respect to $\alpha$ parameter.]

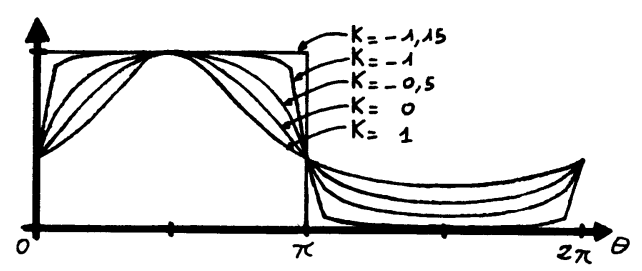

Fig. 15. - Quelques formes d'ondes optimales de courant dans une machine à réluctance variable à variation de perméance sinusoïdale excitée.

[A few optimal current waveforms in an excited R.M. with a sine variation of permeance.]

négatif n'est pas pour autant très important : un exemple est donné figure 16.

L'étude des harmoniques de couple montre que le taux d'harmoniques est, en général, moins élevé dans une machine excitée avec un courant optimisé. On en déduit aussi le nombre de phases intéressant pour la machine. Pour un courant rectangulaire, on choisira $q=3$. Puis ensuite, à mesure que $K$ augmente, il faut augmenter le nombre de phases $q$ pour une annulation suffisante des harmoniques. A titre de comparaison, on donne figure 17 la décomposition en série de Fourier du couple obtenu en linéaire $(K=1)$ pour une onde de courant sinusoïdale. La perte relative de couple correspondant à cette forme d'onde non optimale est de $15 \%$, mais il est alors possible d'éliminer toute ondulation de couple avec une machine à quatre phases. Il apparait ici que l'on puisse choisir entre obtenir la puissance maximale de la machine avec une ondulation de couple ou dégrader les performances de la machine et sortir la puissance avec un couple sans ondulation.

Pour $\alpha=0, K$ ne peut plus être positif, la maximisation amènerait alors à un courant moyen infini. Pour $K<0$, on peut représenter la courbe donnant le rapport $\langle c\rangle / J^{\prime 2}$ en fonction de $J^{\prime} / J_{\mathrm{S}}$ qui est encore égal à $J_{\mathrm{A}} / J_{\mathrm{S}}$. Ce réseau de courbes est donné figure 18. 


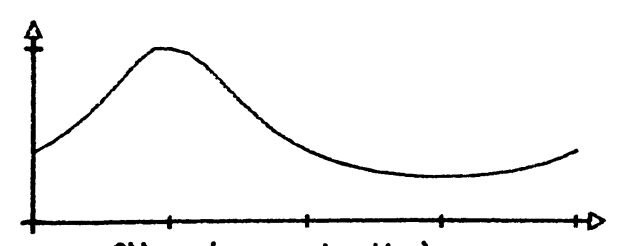

Allure du courant optial

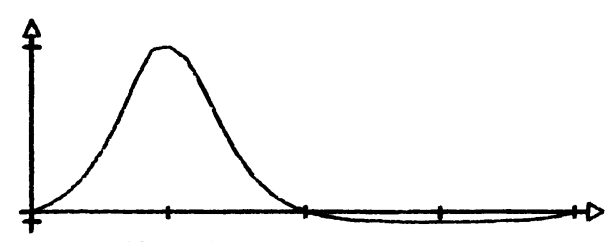

Allure de couple correspondent

Fig. 16.

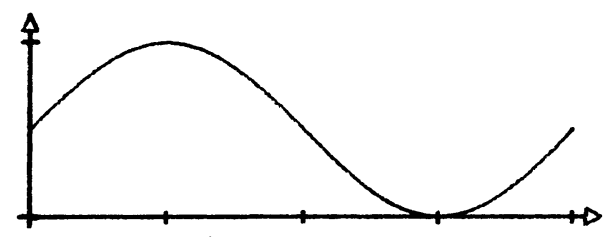

Allure de courant

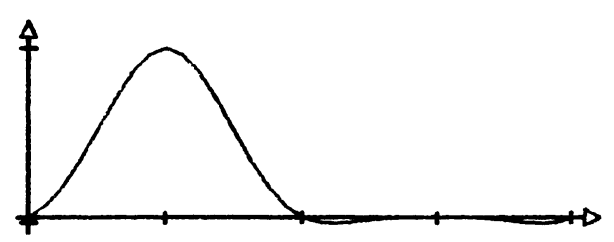

Allure in couple correspondant

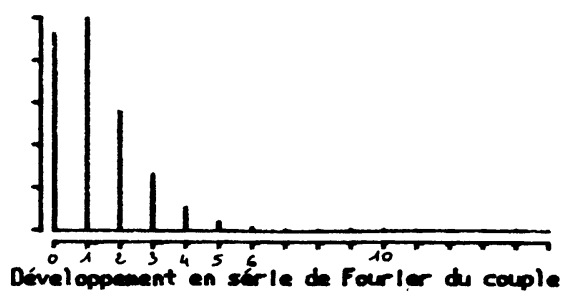

$$
\begin{gathered}
K=1 \\
J^{\prime} / J_{\mathbf{S}}=1 \\
\text { ALPHA }=0,2
\end{gathered}
$$

$$
\begin{gathered}
K=1 \\
J^{\prime} / J_{S}=1 \\
\text { ALPHA }=0,2
\end{gathered}
$$

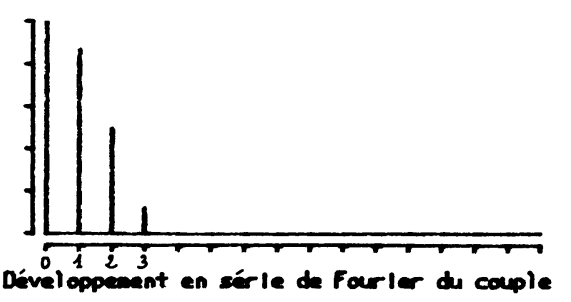

Fig. 17.

On y a aussi représenté, à la même échelle, le réseau de courbes obtenu avec le courant optimal dans une machine à réluctance variable non excitée. On y voit le gain important qu'apporte une excitation " gratuite » et ce d'autant plus que la partie alternative du courant est faible. Cela provient du fait que l'excitation continue permet de polariser la machine à l'endroit où les ampères-tours alternatifs sont le mieux utilisés. C'est le cas d'une polarisation à $J_{\mathrm{S}}$ quand $J_{\mathrm{A}}$ tend vers 0 . Par contre, dans la machine non excitée, les faibles ampères-tours alternatifs utilisent la partie proche de l'origine du cycle $\phi(j)$, ce qui est nettement moins intéressant. A la limite opposée, quand on utilise tout le cycle, le couple moyen est le même, mais, pour une machine excitée, seule la partie alternative des ampères-tours est à fournir par le convertisseur d'alimentation. Ce dernier est donc de taille inférieure à celui qui devra alimenter une machine non excitée. Pour un ensemble convertisseur-machine de faible puissance où le coût du convertisseur est prépondérant, il apparaît que déclasser la machine fera diminuer très notablement la taille du convertisseur. Il faut cependant pondérer tout ce qui vient d'être dit par le fait que l'on ne puisse exactement comparer une machine non excitée ou à excitation par électroaimant (qui peuvent être similaires) à une machine à aimants permanents (qui ne peut être de construction parfaitement identique).

5.2 Perméance TRAPÉzoïdale. - Nous reprenons ici le modèle de variation de perméance du paragraphe 4.2.2. Pour $J^{\prime}$ tendant vers 0 , les gains sur le couple en fonction de $\alpha$ sont toujours donnés par la figure 14 . La forme de courant optimale est une onde à trois 


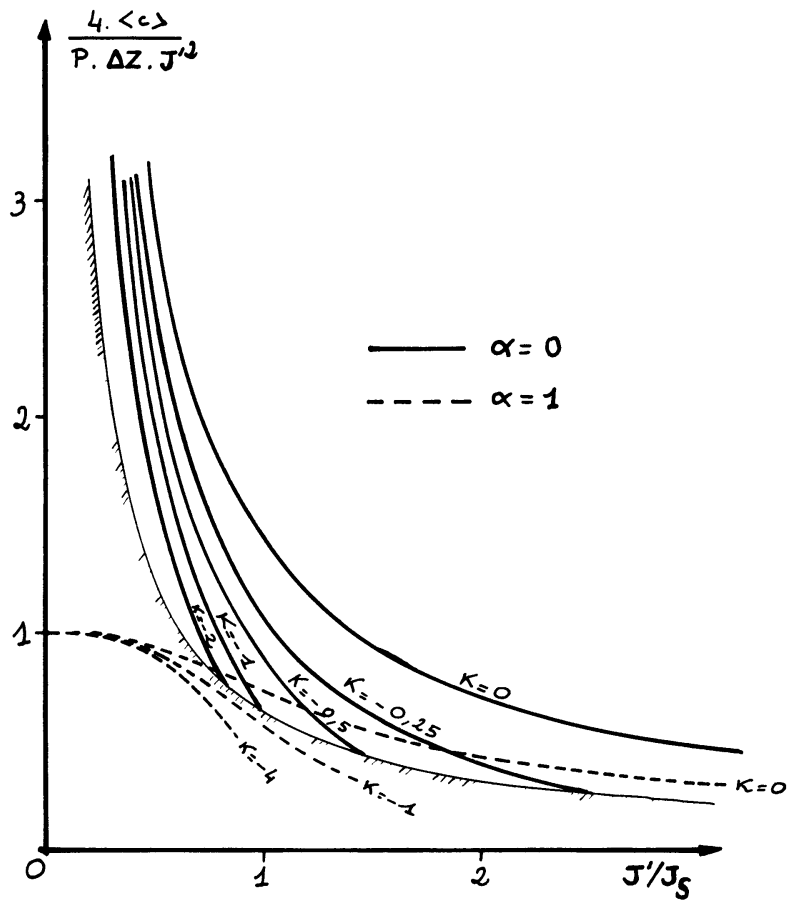

Fig. 18. - Rapport $\langle c\rangle / J^{\prime 2}$ normalisé fonction de $K$ et du courant efficace alternatif normalisé pour $\alpha=0$ et $\alpha=1$.

[Normalized ratio $\langle c\rangle / J^{\prime 2}$ as a function of $K$ and of the RMS value of AC part of current for $\alpha=0$ and $\alpha=1$.]

niveąux. Un premier niveau pour $\mathrm{d} z / \mathrm{d} \theta$ positif, un second pour $\mathrm{d} z / \mathrm{d} \theta$ négatif et un troisième (éventuellement en deux segments) pour $\mathrm{d} z / \mathrm{d} \theta$ nul. Le couple correspondant a, par conséquent, lui aussi une forme à trois niveaux. Le premier niveau du courant correspond à un niveau de couple positif; le second à un niveau de couple négatif; le troisième à un couple nul. En appelant $\Delta \theta$ (Fig. 12) l'angle pendant lequel la variation de perméance est positive (ou négative), on peut obtenir une annulation complète de l'ondulation du couple en choisissant une machine $q$-phasée avec $\Delta \theta=2 \pi / q$.

\section{Conclusions sur la recherche de la forme d'onde optimale du courant d'alimentation.}

Les quelques exemples que nous venons d'exposer montrent qu'il n'y a pas de forme d'onde de courant optimale absolue. Cette forme d'onde optimale dépend tout d'abord du critère d'optimisation choisi, puis de la machine à alimenter. Les calculs précédents ont permis de déterminer l'allure de la forme d'onde optimale dans un certain nombre de cas particuliers.

Dans le cas où l'on désire optimiser le rapport $\langle c\rangle / J_{\mathrm{M}}^{2}$, les résultats sont identiques quelle que soit la machine (forme de perméance, excitation, ...). Il faut utiliser une onde de courant rectangulaire. Notons que l'excitation d'une machine permet de mieux utiliser les ampères-tours alternatifs et d'augmenter ainsi le couple moyen pour une onde de courant alternatif donnée.
Le choix du rapport $\langle c\rangle / J^{2}$ comme critère d'optimisation amène une forme d'onde optimale qui évolue entre l'impulsion de Dirac (machine linéaire non excitée à variation de perméance sinusoïdale) et une forme quasi sinusoïdale (machine saturée excitée à variance de perméance sinusoïdale) en passant par une forme rectangulaire (machine à perméance trapézoïdale). Des formes d'ondes proches de ces formes optimales ont été obtenues par le convertisseur décrit dans l'annexe II. De plus, grâce au programme d'optimisation (décrit en annexe I) mis au point, il est toujours possible de connaître la forme optimale $\mathrm{du}$ courant d'alimentation maximisant le couple moyen obtenu pour un courant efficace donné quelles que soient les variations de perménance de la machine.

On peut aussi choisir d'autres critères d'optimisation comme la minimisation de l'ondulation de couple ou le choix du nombre de phases. Ces deux critères sont souvent liés et on peut minimiser ou même annuler le couple pulsatoire par la combinaison d'une machine $q$-phasée et d'une forme de courant minimisant ou annulant les harmoniques de rang multiple de $q$. A ce point de vue, les machines à variation trapézoïdale de perméance permettent plus facilement d'obtenir une annulation rigoureuse du couple pulsatoire.

Enfin, le travail qui vient d'être exposé concerne essentiellement la maximisation du couple moyen fourni par la machine pour des critères portant sur le courant. Il n'a pas été tenu compte de la tension, ni de ce fait, de la puissance de dimensionnement du convertisseur. C'est sur ce critère d'optimisation que se poursuit actuellement ce travail de recherche.

\section{Annexe I.}

Programme D'optimisation SPÉCIAlisé. - Les temps de calculs des programmes traditionnels d'optimisation sont souvent importants car les méthodes utilisées demandent de recalculer à chaque pas la variable à optimiser, les contraintes et déterminent le point le plus éloigné de la cible. Pour obtenir les mêmes résultats en un temps beaucoup plus court, nous avons été amené à concevoir une méthode d'optimisation plus spécifique mais largement plus rapide. Le programme résultant « tourne » alors sur microordinateur pourtant nettement plus limité en possibilités (temps de cycle et mémoire).

Exposons le problème et la solution choisie.

Il s'agit d'optimiser une fonction de $E$ variables. Ces variables représentent $E$ échantillons du courant sur une période de fonctionnement (éventuellement sur $1 / 2$ ou $1 / 4$ de période en tenant compte de symétries et de considérations physiques).

La fonction à maximiser est :

$$
\sum_{e=1}^{E} c\left(x_{e}\right) \quad \text { à } \sum_{e=1}^{E} x_{e}^{2} \text { donné }
$$


Le programme (organigramme Fig. A1) initialise tout d'abord les $x_{e}$ de manière à ce que $\sum x_{e}^{2}$ soit égal au carré du courant efficace choisi. On calcule alors le couple moyen $\sum c\left(x_{e}\right)$ correspondant.

Soit alors un couple d'échantillons $\left(x_{e_{1}}, x_{e_{2}}\right.$ avec $\left.e_{1} \neq e_{2}\right)$. On fait subir un accroissement à l'un des deux échantillons (par exemple : $x_{e_{1}} \rightarrow x_{e_{1}}^{\prime}$ ). On recalcule la nouvelle valeur du deuxième échantillon $x_{e_{2}}^{\prime}$ de manière à ce que la somme $\sum x_{e}^{2}$ reste constante.
Dans le cas de la machine à un enroulement, on a immédiatement :

$$
x_{e_{2}}^{\prime 2}=x_{e_{2}}^{2}+x_{e_{1}}^{2}-x_{e_{1}}^{\prime 2} .
$$

Pour éviter de recalculer la somme complète à chaque pas (temps proportionnel à $E$ ), on procède par différence entre carrés des anciennes et nouvelles valeurs des seuls échantillons modifiés (gain de temps

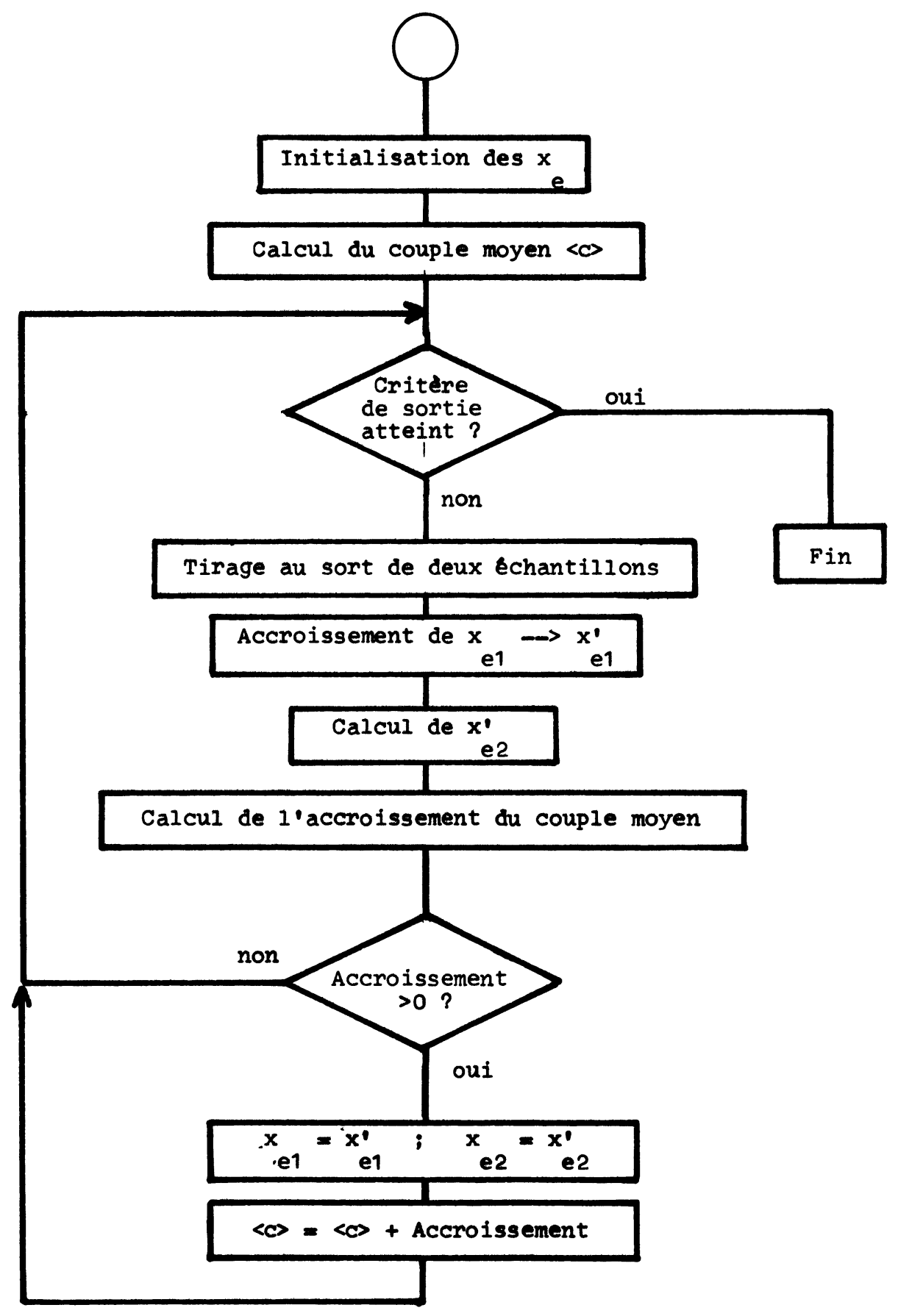

Fig. A.1. - Organigramme simplifié du programme d'optimisation.

[Simplified flow-chart of the optimization programme.] 
de calcul $E / 3)$. On calcule l'accroissement de couple correspondant. Si ce dernier est positif, on remplace les anciennes valeurs des échantillons par les nouvelles. On réajuste le couple moyen, puis on réitère l'opération sur un autre couple $\left(e_{1}, e_{2}\right)$. Si l'accroissement du couple est négatif, on recommence l'opération sur les mêmes échantillons mais en permutant leur rôle.

Dans le cas de la machine à deux enroulements et à ampères-tours pondérés, la fonction de coût $J^{\prime 2}$ est alors :

$$
J^{\prime 2}=\frac{1}{E} \sum_{e=1}^{E} x_{e}^{2}+\frac{\alpha-1}{E^{2}} \cdot\left(\sum_{e=1}^{E} x_{e}\right)^{2} .
$$

Elle peut encore s'écrire après avoir extrait $x_{e_{1}}$ et $x_{e_{2}}$ :

$$
\begin{aligned}
J^{\prime 2}=\frac{1}{E}\left(x_{e_{1}}^{2}\right. & \left.+x_{e_{2}}^{2}+\sum_{e=3}^{E} x_{e}^{2}\right)+ \\
& +\frac{(\alpha-1)}{E^{2}}\left(x_{e_{1}}+x_{e_{2}}+\sum_{e=3}^{E} x_{e}\right)^{2} .
\end{aligned}
$$

Or, $J^{\prime 2}$ doit être invariant en changeant $x_{e_{1}}$ et $x_{e_{2}}$ respectivement en $x_{e_{1}}^{\prime}$ et $x_{e_{2}}^{\prime}$. En se donnant $x_{e_{1}}$ et $x_{e_{2}}$ et une nouvelle valeur $x_{e_{1}}^{\prime}, x_{e_{2}}^{\prime}$ est solution de l'équation du second degré suivante :

$$
\begin{aligned}
\left(1+\frac{\alpha-1}{E}\right) \cdot & x_{e_{1}}^{\prime 2}+2 \cdot \frac{\alpha-1}{E} \cdot\left(x_{e_{2}}^{\prime}+\sum_{e=1}^{E} x_{e}-x_{e_{1}}-x_{e_{2}}\right) \cdot x_{e_{1}}^{\prime}+ \\
& +\frac{\alpha-1}{E} \cdot\left(x_{e_{2}}^{\prime}-x_{e_{1}}-x_{e_{2}}+2 * \sum_{e=1}^{E} x_{e}\right) \cdot\left(x_{e_{2}}^{\prime}-x_{e_{1}}-x_{e_{2}}\right)+x_{e_{2}}^{\prime 2}-x_{e_{2}}^{2}-x_{e_{1}}^{2}=0 .
\end{aligned}
$$

En mémorisant à chaque pas, outre les valeurs de $x_{e}$, les valeurs de $x_{e}^{2}$ et de $\sum x_{e}$, le calcul des coefficients puis des racines de l'équation du second degré n'amène pas un temps de calcul prohibitif (12 add./soustr. 10 mult./div., 1 rac. carrée). On doit ensuite vérifier si les deux solutions obtenues sont compatibles avec les contraintes inhérentes au problème à résoudre. Enfin, l'examen du signe de $c\left(x_{e_{1}}^{\prime}, \theta_{1}\right)-c\left(x_{e_{1}}, \theta_{1}\right)+$ $c\left(x_{e_{2}}^{\prime}, \theta_{2}\right)-c\left(x_{e_{2}}, \theta_{2}\right)$ indique si la nouvelle valeur $x_{e_{1}}^{\prime}$ doit ou non être prise en compte. Dans le premier cas, on mémorise les deux nouvelles valeurs ainsi que leurs carrés. On réactualise aussi la somme des $x_{e}$ et la somme des couples instantanés. Sinon, on laisse les anciennes valeurs. Dans tous les cas, on réitère le processus jusqu'à ce que le couple total soit stationnaire.

Le choix des échantillons est confié à un générateur de nombres aléatoires de manière à ne favoriser aucun échantillon. Statistiquement, chaque échantillon est ainsi comparé à tous les autres au bout de $E^{2} / 2$ essais environ. Le taux de croissance (et de décroissance) est variable et diminue en fonction du nombre d'essais effectués. On arrête l'algorithme quand l'accroissement relatif du couple moyen est inférieur à une limite donnée $\left(10^{-5}\right.$ à $\left.10^{-6}\right)$ après un nombre d'itération fixé. De plus, l'évolution de la forme du courant est visualisée et modifiée au fur et à mesure de l'avancement du calcul. La longueur moyenne de ce programme a permis d'utiliser le BASIC pour ses qualités indéniables de facilité de mise au point (langage interprété, semi-compilé et compilé). Grâce à une programmation suffisamment structurée, le programme peut être modifié extrêmement rapidement pour être adapté aux divers modèles de machines, pour tenir compte des symétries ou pour modifier les sorties des résultats. Le fonctionnement et l'interactivité toujours présente font de ce programme un outil rapide et efficace pour la détermination du maximum du rapport $\langle c\rangle / J^{2}$ et de la courbe $j(\theta)$ optimale.

\section{Annexe II.}

RÉALISATION D'UN CONVERTISSEUR. - L'étude précédente est issue du désir d'alimenter un prototype de machine à réluctance variable pour connaître ces limites de performances. Les principales caractéristiques de ce prototype à variation de perméance sinusoïdale sont données dans [2]. Nous avons été ainsi amené à alimenter cette machine par des formes d'ondes de courant proche du créneau et de l'impulsion de Dirac. Le niveau de puissance envisagé imposant l'utilisation de thyristors, il nous a fallu concevoir un convertisseur original pour généraliser de telles formes d'ondes. En fait, les taux de variation appliquée à la machine et donc par les tensions appliquées aux composants semiconducteurs. Cela implique de dégrader légèrement les formes d'ondes optimales, en particulier les fronts de montée et de descente. D'autre part, nous désirons un seul convertisseur de structure très simple pour générer les deux formes d'onde désirées. Le schéma de ce convertisseur est donné figure A.2.

Deux formes d'ondes typiques obtenues sont données sur les photos A.3 et A.4. On remarque en particulier sur la photo A. 3 la reconstitution numérique de la variation de perméance.

\section{Remerciements.}

Je tiens à remercier Monsieur le Professeur Rioux qui m'a proposé ce sujet de travail et qui m'a permis de le valider expérimentalement en m'accueillant dans son laboratoire. 

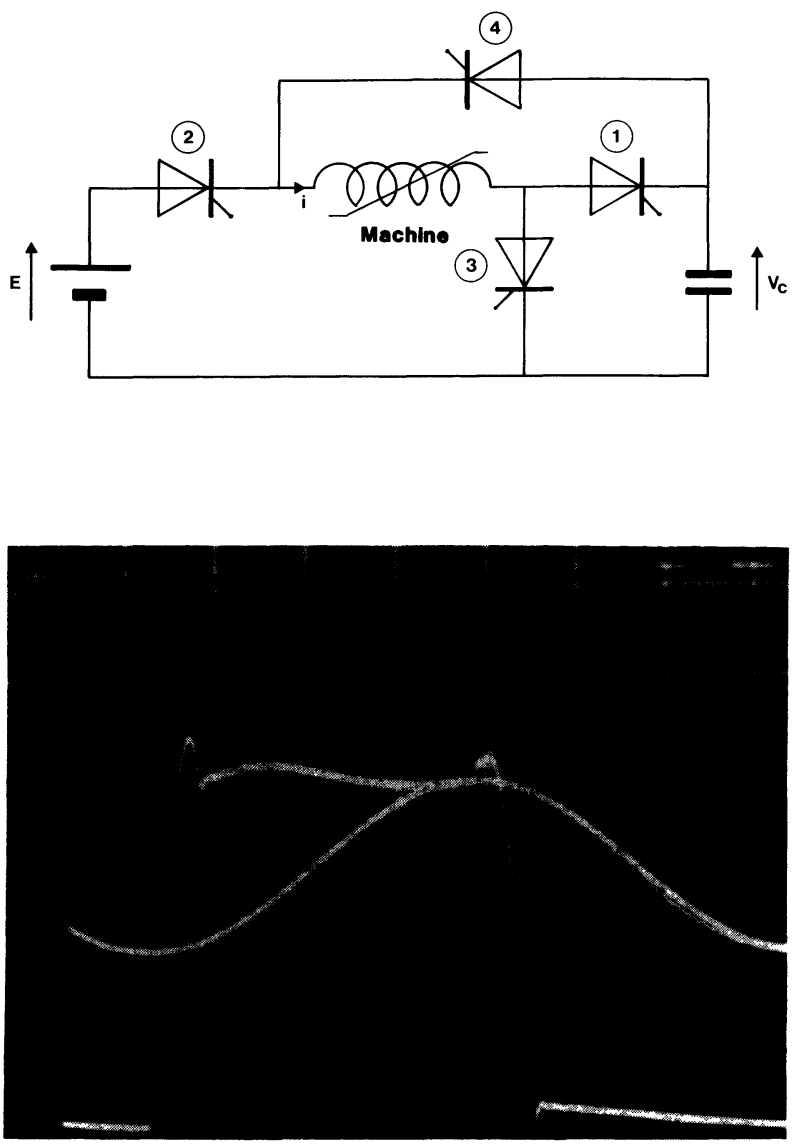

Fig. A.3. - Courant pseudo-rectangulaire obtenu (20 A/ div. ; $0,5 \mathrm{~ms} /$ div.).

[Pseudo square wave supplied by the converter.]
Fig. A.2. - Schéma du convertisseur. [Converter scheme.]

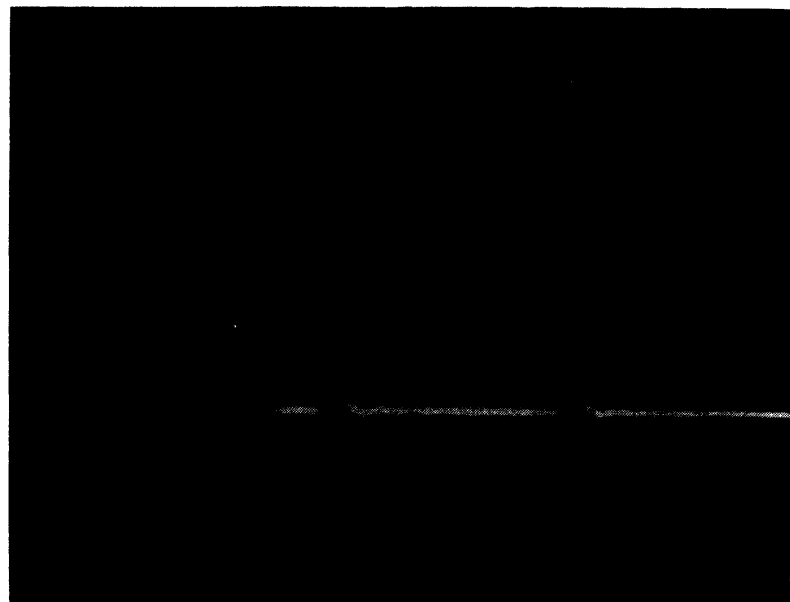

Fig. A.4. - Courant impulsonnel obtenu (20 A/div. ; $10 \mathrm{~ms} /$ div.).

[Pulsed current supplied by the converter.]

\section{Bibliographie}

[1] Rioux, C., «Aspects préliminaires de la théorie des machines électriques comportant des matériaux ferro-magnétiques ", Rev. Phys. Appl. 15 (1980) $1505-1515$.

[2] GOYET, R. and Bleiss, C. A., « Experimental study of an elementary interleaved disk reluctance machine. " ICEM 80 Athens, 427-434.

[3] Bastos, J. P., Goyet, R. et Lucidarme, J., « Performances intrinsèques des machines à réluctance variable à disques imbriqués ", Rev. Phys. Appl. 15 (1980) 45-54.

[4] Bastos, J. P., «L'utilisation de plots de forme trapézoïdale dans les machines à réluctance variable ", Rev. Phys. Appl. 17 (1982) 693-700.

[5] UNNEWEHR, L. E., "Series-commutated SCR controllers for variable speed reluctance motor drives ", PESC 73 Record, 180-191.

[6] Stephenson, P. J., Stephenson, J. M., Blenkinsop, P. T., CoRdA, J. and Fulton, N. N., « Variablespeed switched reluctance motors », IEEE Proc.,
Vol. 127, Pt B, No 4 (1980) 253-265.

[7] Dinov, V. R. and Lazarov, V. D., « Variable reluctance motor with a disc rotor with electronic commutation ", ICEM 80 Athens, 1215-1223.

[8] FAuCher, J., «Contribution à l'étude des machines à réluctance variable à commutation électronique ", Thèse d'état. Toulouse (1981).

[9] Byrne, J., V. and McMullin, M. F., "A saturable reluctance motor investigated as a spindle in the kW range ", ICEM 82 Budapest, 266-269.

[10] ANDRÉ, P., Kauffmann, J. M., LhOTE, F. et Talllard, J. P., «Les robots». Tome 4 : Constituants technologiques. Ed. Hermès, Chap. 4.

[11] Salame, E., Goyet, G., Lucidarme, J., Quichaud, G. et Rioux, C., "Etude d'un moteur à réluctance variable à disques imbriqués alimenté à tension constante ", Rev. Phys. Appl. 19 (1984) 533-544.

[12] Meisel, J., « Principles of electromechanical-energy conversion ", MacGraw-Hill Co. (1966). 